\title{
The evolution and simulation of the outburst from XZ Tauri - A possible EXor?
}

\author{
D. Coffey ${ }^{1}$, T. P. Downes ${ }^{2}$, and T. P. Ray ${ }^{1}$ \\ 1 School of Cosmic Physics, Dublin Institute for Advanced Studies, 5 Merrion Square, Dublin 2, Ireland \\ 2 Dublin City University, Dublin 9, Ireland
}

Received 12 September 2003 / Accepted 19 February 2004

\begin{abstract}
We report on multi-epoch HST/WFPC2 images of the XZ Tauri binary, and its outflow, covering the period from 1995 to 2001. Data from 1995 to 1998 have already been published in the literature. Additional images, from 1999, 2000 and 2001 are presented here. These reveal not only further dynamical and morphological evolution of the XZ Tauri outflow but also that the suspected outflow source, XZ Tauri North, has flared in EXor-type fashion. In particular our proper motion studies suggests that the recently discovered bubble-like shock, driven by the the XZ Tauri outflow, is slowing down (its tangential velocity decreasing from $146 \mathrm{~km} \mathrm{~s}^{-1}$ to $117 \mathrm{~km} \mathrm{~s}^{-1}$ ). We also present simulations of the outflow itself, with plausible ambient and outflow parameters, that appear to reproduce not only the dynamical evolution of the flow, but also its shape and emission line luminosity.
\end{abstract}

Key words. ISM: Herbig-Haro objects - ISM: jets and outflows - stars: pre-main sequence - stars: formation stars: individual: XZ Tau - stars: binaries (including multiple): close

\section{Introduction}

$\mathrm{XZ}$ Tau is a classical T Tauri binary system with a separation of 0.3 (Haas et al. 1990) and is located in the well known Lynds 1551 star-forming region some $140 \mathrm{pc}$ away (Elias 1978). The system was first found to have an associated Herbig-Haro (HH) outflow through ground-based CCD imaging and spectroscopy (Mundt et al. 1988; Mundt et al. 1990). These early observations revealed a bipolar optical flow that could be traced to at least $10^{\prime \prime}$ on either side of the binary at a position angle of $15^{\circ}$. The first Hubble Space Telescope (HST) Wide Field Planetary Camera 2 (WFPC2) images of XZ Tauri taken in 1995 show a bubble of emission nebulosity extending 4 " to the north of the system (Krist et al. 1997, hereafter K97). Further images, taken 3 years later, show dramatic structural changes as the bubble expanded and altered from being centre-brightened to limb-brightened, suggesting the formation of a HH bowshock (Krist et al. 1999, hereafter K99).

Ground-based photometry of XZ Tau from 1962 to 1981 (Herbst et al. 1994) has shown variations, of almost two magnitudes in the $\mathrm{V}$ band, for the binary as a whole. Such variations are common amongst young stellar objects (YSOs). Of the two components, the southern one has been, at least until recently, optically brighter and thought to be the more evolved star. Its companion, however, dominates at infra-red wavelengths and is probably of higher overall luminosity (Haas et al. 1990).

Send offprint requests to: D. Coffey, e-mail: dac@cp.dias.ie
Recent Faint Object Spectrograph (FOS) observations, however, unexpectedly found the northern component to be optically brighter (White \& Ghez 2001, hereafter WG01), a result that we will discuss further in the light of our findings. For this system therefore it seems more appropriate not to use the terms primary and secondary but instead we will adopt the nomenclature, used in K97, of XZ Tau North and South.

We report here analysis of further HST Archive WFPC2 images of the XZ Tau system and outflow from 1999, 2000 and 2001. These data show not only ongoing changes in the outflow but a dramatic brightening of XZ Tau North in the optical suggesting that it may be an EXor. We also simulate the outflow in an attempt to reproduce its dynamical and morphological evolution.

\section{Data}

High resolution archival WFPC2 images of XZ Tauri were obtained for 3 consecutive epochs: 1999 February 3; 2000 February 6; and 2001 February 10. The binary was at the same approximate location on the Planetary Camera (spatial sampling $=00^{\prime} 04555$ pixel $\left.^{-1}\right)$ for all frames. Four filters were used: $\mathrm{H} \alpha(F 656 N)$; [SII] $(F 673 N)$; $R$-band $(F 675 W)$ and $I$-band $(F 814 W)$. The frequency and duration of the short exposures for each filter were: $1 \times 120 \mathrm{~s} ; 1 \times 180 \mathrm{~s} ; 2 \times 6 \mathrm{~s}$; and $2 \times 6 \mathrm{~s}$ respectively. Two long exposures for each filter of $1000 \mathrm{~s}$ were also extracted from the HST Archive, all of which were saturated at the location of XZ Tauri. No short exposures were made for the $I$-band filter in the final year. 

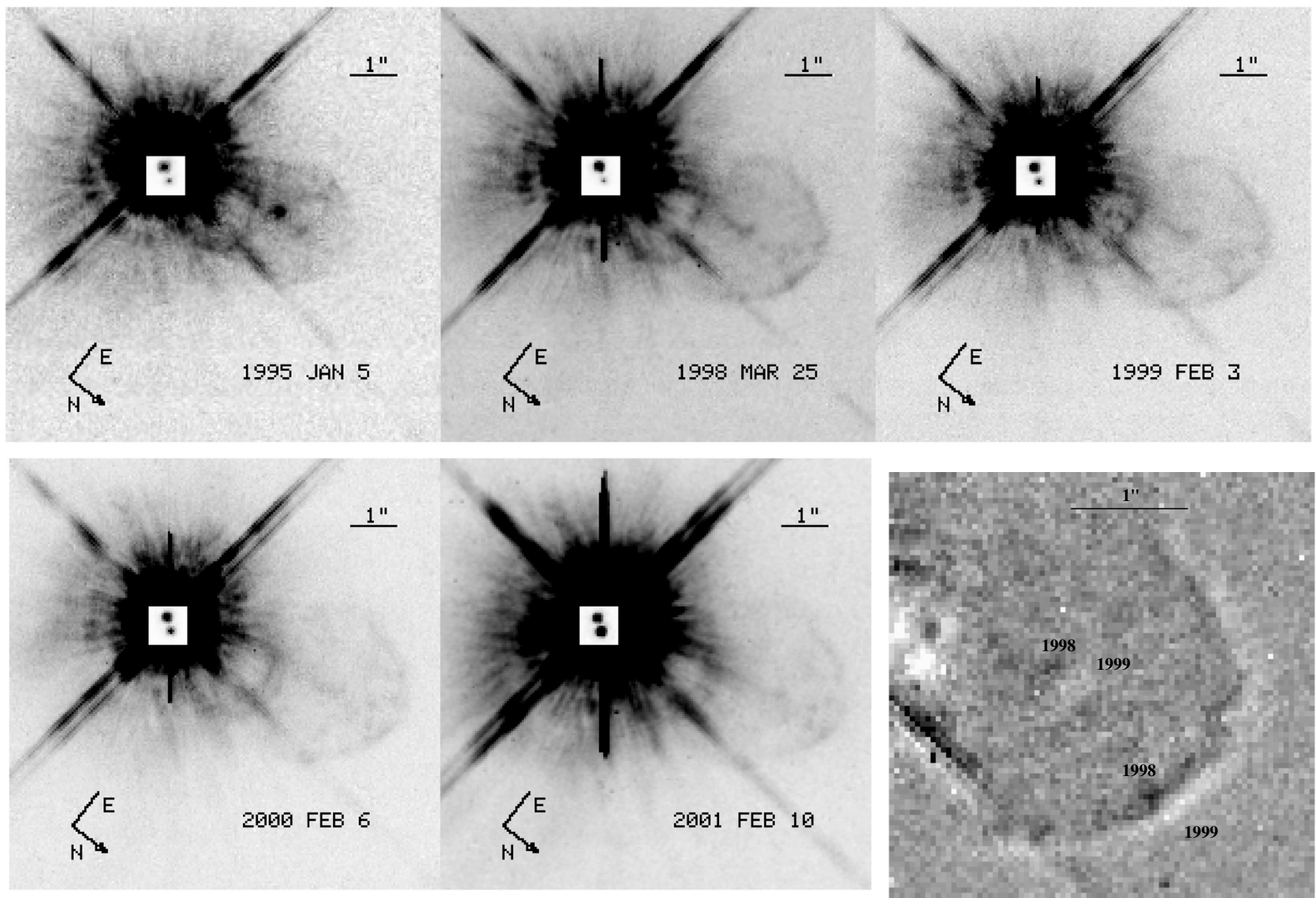

Fig. 1. HST/WFPC2 F675W (R-band) images of XZ Tau and its outflow on: 1995 Jan. 5; 1998 Mar. 25; 1999 Feb. 3; 2000 Feb. 6; and 2001 Feb. 10. The short exposure image of the binary is superimposed on the long exposure image in each case. The bottom right frame is an $R$-band difference image 1999-1998 showing proper motion in the jet and the outer shock.

Previously published WFPC2 archival data (K99) for XZ Tauri facilitated proper motion measurements. These data comprised images from 1995 January 5 and 1998 March 25. The 1995 images used were $2 \times 600$ s exposures in the $R$-band and $1 \times 3 \mathrm{~s}$ exposure in the $I$-band. The latter allowed us to determine the stellar positions, as no $R$-band short exposures were taken and the long exposures were saturated in the vicinity of the star. The 1998 images were taken in the $R$-band only: $2 \times 6 \mathrm{~s}$ and $2 \times 1100 \mathrm{~s}$ exposures. All frames were processed through the standard HST pipeline and each set of double exposures was combined to eliminate cosmic rays.

The accumulated data yielded high resolution images of XZ Tau spanning a total of 6 years. We analyse these data in Sect. 3, under the two headings: outflow structure, proper motions and [SII] luminosity; and stellar astrometry and photometry.

\section{Results}

\subsection{Outflow structure, proper motions and [SII] luminosity}

The Planetary Camera images for each year were aligned and their orientation corrected where necessary. A montage of
$R$-band images, covering the full timespan, is shown in Fig. 1 . As previously observed (K99), there appears to be two edgebrightened "bubbles", i.e. inner and outer shocks, similar to those seen with HST close to DG Tau (Bacciotti et al. 2000). By 2001, the rapidly fading outer shock reached a distance of approximately $800 \mathrm{AU}$ from the binary, while the inner shock has travelled approximately half as far.

Although remarkably bright in 1995 , the knots in the XZ Tau jet appear very faint by 2001 . The jet's PA is around $15^{\circ}$ in line with the major axis of the elongated outer bubble and the known extended optical outflow. The knots however are not in a perfectly straight line as some deviation is evident and reminiscent of similar behaviour seen in flows like the HH34 jet close to its source (Reipurth et al. 2002).

Significant proper motions were observed as the bubble expanded away from XZ Tau. Tangential velocities for the central jet knot and outer shock (see for example the bottom right "difference" frame in Fig. 1) were obtained from the long $F 675 \mathrm{~W}$ exposures. The latter were used since they include the main $\mathrm{HH}$ emission lines, i.e. [OI] $\lambda \lambda 6300,6363, \mathrm{H} \alpha$ and $[\mathrm{SII}] \lambda \lambda 6717$, 6731 . The distance to the binary was assumed to be $140 \mathrm{pc}$ as in K99. In Table 1 we list the derived tangential velocities as a function of epoch for the outer shock and an average velocity for the jet. Longitudinal sizes and speeds were calculated along 
Table 1. Projected sizes and speeds of the XZ Tauri outer bowshock (and an average speed of the jet knot) as it evolves. Distances are quoted for the latter date of the observation interval. Errors are at the three sigma level.

\begin{tabular}{lcccc}
\hline \hline $\begin{array}{l}\text { Observation } \\
\text { date/interval }\end{array}$ & $\begin{array}{c}\text { Distance } \\
\text { from source } \\
\text { (AU) }\end{array}$ & $\begin{array}{c}\text { Bubble } \\
\text { width } \\
\text { (AU) }\end{array}$ & $\begin{array}{c}\text { Longitudinal } \\
\text { speed } \\
\left(\mathrm{km} \mathrm{s}^{-1}\right)\end{array}$ & $\begin{array}{c}\text { Transverse } \\
\text { speed } \\
\left(\mathrm{km} \mathrm{s}^{-1}\right)\end{array}$ \\
\hline 1995 Jan. 5 & $598 \pm 16$ & $336 \pm 25$ & - & - \\
1995 Jan. 5 to 1998 Mar. 25 & $697 \pm 10$ & $405 \pm 14$ & $146 \pm 28$ & $101 \pm 42$ \\
1998 Mar. 25 to 1999 Feb. 3 & $721 \pm 9$ & $423 \pm 5$ & $130 \pm 73$ & $105 \pm 83$ \\
1999 Feb. 3 to 2000 Feb. 6 & $746 \pm 11$ & - & $119 \pm 65$ & - \\
2000 Feb. 6 to 2001 Feb. 10 & $771 \pm 10$ & - & $117 \pm 69$ & - \\
1998 Mar. 25 to 2001 Feb. 10 & - & - & $130 \pm 84$ (jet) & - \\
\hline
\end{tabular}

the jet axis $\left(\mathrm{PA} \sim 15^{\circ}\right)$. Transverse sizes and speeds were calculated perpendicular to the jet axis at the bubble's widest point, (specifically, the point where the source-to-shock line forms an angle of $20^{\circ}$ with the jet axis in the 1995 image). As the shock expands it grows fainter and so its width is not given for later observations. The central knot also grows very faint, especially at later epochs, so we prefer to quote an average for the jet speed. Finally, although the inner shock or bubble was seen to expand, changes from year to year proved difficult to trace and so no tangential velocity is given here.

From Table 1 it is clear that we see a deceleration of the outer shock. Although the errors appear large, if we consider the interval 1998-2001 we obtain a longitudinal speed of $121( \pm 24) \mathrm{km} \mathrm{s}^{-1}$ (cf. $146( \pm 28) \mathrm{km} \mathrm{s}^{-1}$ from 1995 to 1998$)$ suggesting the shock front is decelerating. Simulations were carried out in order to model the observations using plausible jet and ambient medium parameters (see Sect. 3.3 below). Observerd speeds are as expected in order to maintain the observed low aspect ratio of the bubble, and are in the same region as those given by the simulation, (see Table 4 below). As a further check the simulated and observed [SII] $\lambda \lambda 6317,6731$ luminosities were compared. The latter was found from the $2001 F 631 N$ images to be $2.5( \pm 3.6) \times 10^{28} \mathrm{erg} \mathrm{s}^{-1}$, excluding the inner shock region which is contaminated with diffraction spikes and nebulosity near the star. If we assume an extinction of $A_{\mathrm{v}} \sim 1.39$ towards the bubble, i.e. the same as towards the binary (WG01), the intrinsic luminosity increases to $\sim 4.3 \times 10^{28} \mathrm{erg} \mathrm{s}^{-1}$.

\subsection{Stellar astrometry and photometry}

Using the short $R$ band exposures, the separation and position angle of the binary were determined for the different epochs and are listed in Table 2. Within errors, no changes were detected in the separation of the XZ Tau binary although an average decrease of $0.75^{\circ} \mathrm{yr}^{-1}$ was observed in its position angle, a value somewhat higher than derived by K99 based on earlier HST data alone (of $0.5^{\circ} \mathrm{yr}^{-1}$ ) but at the same time lower than that found by Woitas et al. (2001) using speckle interferometry (of $\sim 1.3^{\circ} \mathrm{yr}^{-1}$ ). Following K99, if we assume a face-on orbit, the total mass for the binary is about $0.3 M_{\odot}$. Although such a value is clearly too low, note that the combined mass is very sensitive to the projection angle. Such a figure is
Table 2. Separation and position angle of the XZ Tau binary. Errors in separation angles are estimated to be \pm 0.005 .

\begin{tabular}{lll}
\hline \hline $\begin{array}{l}\text { Date of } \\
\text { observation }\end{array}$ & $\begin{array}{l}\text { Separation } \\
(\operatorname{arcsec})\end{array}$ & $\begin{array}{l}\text { Position angle } \\
\text { (degrees) }\end{array}$ \\
\hline 1995 Jan. 5 & 0.302 & $146.35 \pm 0.38$ \\
1998 Mar. 25 & 0.299 & $145.50 \pm 0.25$ \\
1999 Feb. 3 & 0.303 & $144.85 \pm 0.26$ \\
2000 Feb. 6 & 0.300 & $143.30 \pm 0.25$ \\
2001 Feb. 10 & 0.298 & $142.38 \pm 0.24$ \\
\hline
\end{tabular}

Table 3. Johnson apparent magnitudes of the XZ Tauri binary. Errors in magnitude of \pm 0.05 were estimated based on the affect of changing aperture size, given that the stellar PSFs are overlapping. The 1995 Jan. $5 R$ band data is from K97 using Tiny Tim PSF fitting to the saturated $R$ band images. The 1997 Mar. 8 data is from GO Programme 6725.

\begin{tabular}{llccc}
\hline \hline Filter & $\begin{array}{l}\text { Observation } \\
\text { date }\end{array}$ & $\begin{array}{c}\text { Flux ratio } \\
\text { South/North }\end{array}$ & $\begin{array}{c}\text { XZ South } \\
\text { magnitude }\end{array}$ & $\begin{array}{c}\text { XZ North } \\
\text { magnitude }\end{array}$ \\
\hline$R$-band & 1995 Jan. 5 & 5.11 & 13.16 & 14.93 \\
& 1997 Mar. 8 & 5.39 & 13.47 & 15.38 \\
& 1998 Mar. 25 & 9.02 & 13.22 & 15.68 \\
& 1999 Feb. 3 & 3.66 & 13.45 & 14.93 \\
& 2000 Feb. 6 & 2.43 & 13.32 & 14.36 \\
& 2001 Feb. 10 & 0.63 & 13.25 & 12.82 \\
$I$-band & & & & \\
& 1995 Jan. 5 & 4.80 & 12.03 & 13.78 \\
& 1997 Mar. 8 & 6.30 & 11.97 & 14.02 \\
& 1999 Feb. 3 & 3.38 & 12.04 & 13.42 \\
& 2000 Feb. 6 & 1.98 & 12.02 & 12.82 \\
\hline
\end{tabular}

certainly lower than the estimates of Hartigan \& Kenyon (2003) and WG01 suggesting a combined mass closer to $1 M_{\odot}$.

Broadband $R$ and $I$ magnitudes were calculated for the binary, using the method outlined in the WFPC2 Data Reduction Handbook and interpolated Johnson offsets appropriate for the spectral types of XZ Tau North and South, i.e. M 2 and M 3.5 respectively (Hartigan \& Kenyon 2003). Our results are presented in Table 3 and the $R$-band WFPC2 data were used to plot a light curve for each component, Fig. 2. Over the six years of observations, XZ Tau South although it varies does so by at most 0.3 mag (in $R$ ) and has a mean $R$ magnitude of around 13.5. In contrast, $X Z$ Tau North shows an initial reduction in brightness of about a magnitude in $R$ until 1998 and thereafter 




Fig. 2. Stellar Johnson $R$-band apparent magnitudes of the XZ Tauri binary.

its brightness increases dramatically by around $3 \mathrm{mag}$. This flaring behaviour means that by 2001 XZ Tau North was actually the brighter star. Similar variations are seen in the $I$ band although the data are somewhat more sparse.

The dramatic brightening of XZ Tau North suggests it is an EXor. EXors, named after their prototype EX Lupi, are extreme classical $\mathrm{T}$ Tauri stars that periodically undergo outbursts from the UV to the optical. Although increases by several magnitudes with rise times of up to a few years have been recorded (Herbig 1989) the changes in these YSOs are not as extreme as in FU Orionis stars. EXor spectra, for example, even in outburst continue to resemble $\mathrm{T}$ Tauri stars. The phenomenon is thought to be due to major increases in the underlying disk accretion rate, but the number of EXors known is relatively small. The proposition that XZ Tau North is such a YSO is further strengthened by the HST spectroscopic data of Hartigan \& Kenyon (2003). As with other EXors the spectrum shows not only very strong Balmer lines but also strong $\mathrm{Ca}$ II and moderate $\mathrm{Na}$ I in emission (see, for example, Parsamian et al. 2002). Such strong spectral emission lines coupled with high variability makes XZ Tau North the most likely source of the outflow. Finally, EXor behaviour would explain why the 1996 HST/FOS acquisition of XZ Tauri (WG01) unexpectedly locked onto the wrong (northern) star. WFPC2 images taken in March 1997, as part of HST GO Programme 6725, show XZ Tauri North comparable in brightness with XZ Tauri South at short optical wavelengths. This also suggests that EXor behaviour may be visible at the shortest wavelengths first.

\subsection{Modelling the outflow}

In this section we present the results of a numerical simulation of the XZ Tauri outflow in order to check our physical interpretation of the observations. The code we use is that described in Downes \& Ray (1999). It is a properly upwinded second order (in time and space), cylindrically symmetric code for solving the inviscid Euler equations. In addition to tracking the hydrodynamic variables (density, velocity and pressure), the code also tracks the ionisation state of hydrogen, without the assumption of ionisation equilibrium. It also has the capacity to track the number density of $\mathrm{H}_{2}$, but this number density was set to zero for these simulations.

\subsubsection{Initial conditions}

The gas is taken to be one of solar abundances. The initial ambient density is assumed to be $100 \mathrm{~cm}^{-3}$ close to the source, rising to a value of $600 \mathrm{~cm}^{-3}$ at a distance of $1.25 \times 10^{16} \mathrm{~cm}$ from the source, (see below for a discussion of why this behaviour is assumed). The ambient pressure was taken to be uniform, giving a temperature of $10^{3} \mathrm{~K}$ close to the source, and dropping to approximately $160 \mathrm{~K}$ at the rise in ambient density. The resolution was set to $1 \times 10^{13} \mathrm{~cm}$ in both the radial and poloidal (outflow) directions ( $r$ and $z$ respectively), and the total grid size was $450 \times 1500$. To estimate the mass flux in the outflow, we assumed approximately $2 \%$ of the accreted mass ultimately ends up in the outflow (Hartigan et al. 1995). A mass accretion rate for XZ Tau North of $1 \times 10^{-7} M_{\odot} \mathrm{yr}^{-1}$ was used (Hartigan \& Kenyon 2003) based on recent photospheric veiling measurements.

In order to choose parameters for the outflow we must take the following issues into consideration:

1. the aspect ratio of the outer bowshock is small despite the fact that the outflow is thought to be highly supersonic with respect to the sound speed in the ambient medium;

2. if we assume a constant jet/outflow radius based on the observed inner knots, i.e. $r_{\text {jet }} \sim 2 \times 10^{14} \mathrm{~cm}$, then the inferred mass flux in the outflow would imply a large jet density and specifically one that is much higher than the ambient density. This cannot be the case for the XZ Tauri outflow, as such a highly dense jet would simply plough through the local medium creating a very narrow bowshock, in contradiction with our observations.

One way of explaining the observations is to invoke a moderately collimated wind. A wind with a moderate opening angle will have a density which decreases significantly with distance from the source, and will also have a larger radius with distance. These effects each lead to a broader bowshock, in line with the observed morphology of the system. In addition, if the flow is not in the plane of the sky, the bowshock will appear to have a lower aspect ratio due to projection effects. We assume an angle of $30^{\circ}$ between the plane of the sky and the axis of the outflow.

With these considerations in mind, we set the full opening angle of the wind to be $22^{\circ}$ with an initial diameter (i.e. $F W H M$ in density) of $4 \times 10^{14} \mathrm{~cm}$, or 40 grid cells. The wind density was given a linear profile across the outflow axis, with a density range from $\sim 1800 \mathrm{~cm}^{-3}$ along the jet axis to $\sim 100 \mathrm{~cm}^{-3}$ at the edge, and was chosen so that the total mass flux was $2 \times 10^{-9} M_{\odot} \mathrm{yr}^{-1}$. Hence we are assuming the jet (traced by the knots) does not dominate the dynamics of the system.

The observed tangential speed of the flow is in the range $130-200 \mathrm{~km} \mathrm{~s}^{-1}$ so we choose a constant wind velocity of 
Table 4. Projected sizes and speeds of the simulated XZ Tauri outer bowshock as it evolves. Distances are quoted for the latter year of the age interval. There is a minimum error of $\pm 6 \mathrm{~km} \mathrm{~s}^{-1}$ in the simulation speeds.

\begin{tabular}{|c|c|c|c|c|c|}
\hline $\begin{array}{l}\text { Simulation age } \\
\text { /age interval (years) }\end{array}$ & $\begin{array}{l}\text { Corresponding observation } \\
\text { date/interval }\end{array}$ & $\begin{array}{c}\text { Distance } \\
\text { from source }(\mathrm{AU})\end{array}$ & $\begin{array}{c}\text { Bubble } \\
\text { width (AU) }\end{array}$ & $\begin{array}{l}\text { Longitudinal } \\
\text { speed }\left(\mathrm{km} \mathrm{s}^{-1}\right)\end{array}$ & $\begin{array}{c}\text { Transverse } \\
\text { speed }\left(\mathrm{km} \mathrm{s}^{-1}\right)\end{array}$ \\
\hline 15 & 1995 Jan. 5 & 590 & 288 & - & - \\
\hline $15-18$ & 1995 Jan. 5 to 1998 Mar. 25 & 695 & 360 & 164 & 67 \\
\hline $18-19$ & 1998 Mar. 25 to 1999 Feb. 3 & 722 & 386 & 148 & 63 \\
\hline $19-20$ & 1999 Feb. 3 to 2000 Feb. 6 & 749 & 402 & 129 & 51 \\
\hline $20-21$ & 2000 Feb. 6 to 2001 Feb. 10 & 775 & 420 & 115 & 44 \\
\hline
\end{tabular}



Fig. 3. Log-scale plot of the distribution of density at $t=21 \mathrm{yr}$. The distance scales are in units of $10^{15} \mathrm{~cm}$ and the intensity scales are in units of $\mathrm{g} \mathrm{cm}^{-3}$.

$250 \mathrm{~km} \mathrm{~s}^{-1}$, which will give a tangential velocity for the jet fluid of roughly $216 \mathrm{~km} \mathrm{~s}^{-1}$. Since the bowshock appears to be decelerating significantly (see Table 1), we need some way to slow it down. Turning off the jet will not, on its own, give us the observed deceleration since the momentum density of the material in the cooled shell between the bowshock and jetshock is very large. It was found that the most effective way of decelerating the bowshock was to impose a significant increase in the ambient density. A six-fold increase was found to roughly reproduce the observed velocities.

\subsubsection{Simulation results}

Figure 3 contains a greyscale plot of the distribution of density at $t=21 \mathrm{yr}$, the time at which the simulation roughly matches the 2001 observations. The non-zero opening angle of the jet is clearly noticeable. The bowshock itself has a number of irregularities which probably arise from the Vishniac instability. Another possible source is the Rayleigh-Taylor instability, since the bowshock is decelerating. It can be seen that the bowshock has begun to encounter the rise in the ambient density (see Sect. 3.3.1). This leads to a marked decrease in its speed of advance (see Table 4). The behaviour of the velocity of the outer shock matches that observed reasonably well.

Figure 4 is a simulated [SII] image calculated for the density distribution shown in Fig. 3, assuming an angle to the plane of the sky of $30^{\circ}$. In calculating this we assume that the ionisation fractions state of sulphur can be described by coronal ionisation equilibrium (Arnaud \& Rothenflug 1985) and that the line emission is not in local thermal equilibrium. The emission is plotted on a linear scale (similar to that in Fig. 1). It has been projected onto the sky and convolved with a Gaussian of $F W H M=0 . ' 1$.

It can be seen that, broadly speaking, there is reasonable agreement between the observations and the simulation,

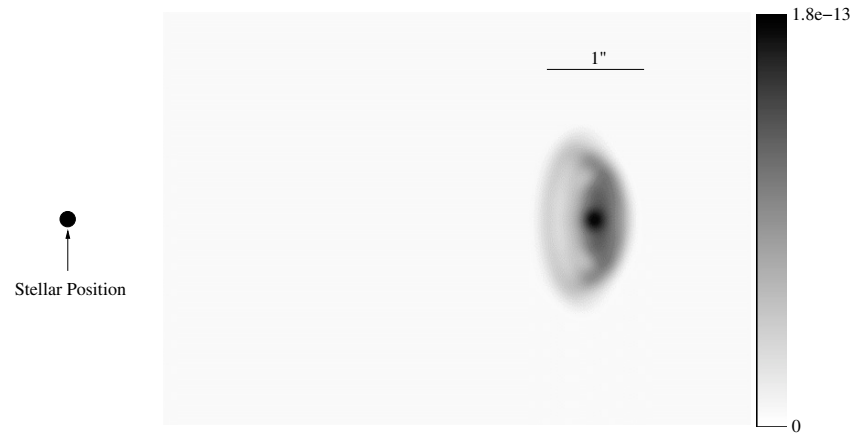

Fig. 4. Simulated [SII] image projected onto the sky (assuming an inclination with respect to the plane of the sky of $30^{\circ}$ ) at $t=21 \mathrm{yr}$ (see text). The intensity scales are in units of $\mathrm{erg} \mathrm{s}^{-1} \mathrm{~cm}^{-2}$.

certainly in terms of the morphology close to the head of the bowshock. The ring-like features arise from the assumption of cylindrical symmetry in the simulation. The emission would, in fact, be expected to be a little more "clumpy" rather than ring-like.

Finally, the total [SII] luminosity calculated from the simulation is $1.1 \times 10^{29} \mathrm{erg} \mathrm{s}^{-1}$. This is in good agreement with that observed (i.e. $4.3( \pm 3.6) \times 10^{28} \mathrm{erg} \mathrm{s}^{-1}$, see Sect. 3.1). We note however that the bowshock apex appears much brighter with respect to the wings than is actually observed. This is most likely due to our use of cylindrical symmetry which results in a focussing of shocked material onto the axis in a way which would be very unlikely to happen in three dimensions.

\section{Conclusions}

Multi-epoch HST/WFPC observations of the XZ Tau binary and its associated outflow have shown considerable changes in the system within only 6 years, from 1995 to 2001 . The presence of two limb-brightened shock fronts is now clearly evident, with a deceleration of the outer shock from $146 \mathrm{~km} \mathrm{~s}^{-1}$ to $117 \mathrm{~km} \mathrm{~s}^{-1}$. Stellar photometry revealed that the suspected source of the outflow, XZ Tau North, has flared in EXor-type fashion increasing in brightness by 3 mag in $R$ between 1998 and 2001. Finally, numerical simulations of the outflow produced reasonable agreement with observation in terms of morphology, dynamical evolution and emission line luminosity, using plausible ambient and outflow parameters. Deceleration by the amount observed, caused by the ambient medium, should have produced a much brighter bowshock apex than that seen. The cause of this discrepancy is not obvious. 
Acknowledgements. We wish to thank the referee, Dr S. Cabrit, for useful comments and suggestions. D.C. and T.P.R. would like to acknowledge support for their research from Enterprise Ireland. This work was carried out as part of the CosmoGrid project, funded under the Programme for Research in Third Level Institutions (PRTLI) administered by the Irish Higher Education Authority under the National Development Plan and with partial support from the European Regional Development Fund.

\section{References}

Arnaud, M., \& Rothenflug, R. 1985, A\&AS, 60, 425

Bacciotti, F., Mundt, R., Ray, T. P., et al. 2000, ApJ, 537, L49

Downes, T. P., \& Ray, T. P. 1999, A\&A, 345, 977

Elias, J. H. 1978, ApJ, 224, 857

Haas, G. M., Leinert, C., \& Zinnecker, H. 1990, A\&A, 230, L1

Hartigan, P., \& Kenyon, S. J. 2003, ApJ, 583, 334
Hartigan, P., Edwards, S., \& Ghandour, L. 1995, ApJ, 452, 736

Herbst, W., Herbst, D. K., Grossman, E. J., \& Weinstein, D. 1994, ApJ, 108, 1906

Herbig, G. H. 1989, Proceedings of the ESO Workshop on Low Mass Star Formation and Pre-main Sequence Objects, 233

Mundt, R., Ray, T. P., \& Buhrke, T. 1988, ApJ, 333, L39

Mundt, R., Buhrke, T., Solf, J., Ray, T. P., \& Raga, A. C. 1990, A\&A, 232, 37

Krist, J. E., Burrows, C. J., Stapelfeldt, K. R., et al. 1997, ApJ, 481, 447 (K97)

Krist, J. E., Stapelfeldt, K. E., Burrows, C. J., et al. 1999, ApJ, 515, L35 (K99)

Parsamian, E. S., Mujica, R., \& Corral, L. 2002, Astrophys., 45, 393

Reipurth B., Heathcote, S., Morse, J., Hartigan, P., \& Bally, J. 2002, ApJ, 123, 362

White, R. J., \& Ghez, A. M. 2001, ApJ, 556, 265 (WG01)

Woitas, J., Köhler, R., \& Leinert, C. 2001, A\&A, 369, 249 